\title{
Intraocular expression and release of high-mobility group box 1 protein in retinal detachment
}

\author{
Noboru Arimura ${ }^{1,2}$, Yuya Ki-i ${ }^{1,2}$, Teruto Hashiguchi², Ko-ichi Kawahara², Kamal K Biswas ${ }^{2}$, Makoto Nakamura ${ }^{3}$, \\ Yasushi Sonoda ${ }^{1}$, Keita Yamakiri ${ }^{1}$, Akiko Okubo $^{1}$, Taiji Sakamoto ${ }^{1}$ and Ikuro Maruyama ${ }^{2}$
}

High-mobility group box 1 (HMGB1) protein is a multifunctional protein, which is mainly present in the nucleus and is released extracellularly by dying cells and/or activated immune cells. Although extracellular HMGB1 is thought to be a typical danger signal of tissue damage and is implicated in diverse diseases, its relevance to ocular diseases is mostly unknown. To determine whether HMGB1 contributes to the pathogenesis of retinal detachment (RD), which involves photoreceptor degeneration, we investigated the expression and release of HMGB1 both in a retinal cell death induced by excessive oxidative stress in vitro and in a rat model of RD-induced photoreceptor degeneration in vivo. In addition, we assessed the vitreous concentrations of HMGB1 and monocyte chemoattractant protein 1 (MCP-1) in human eyes with RD. We also explored the chemotactic activity of recombinant HMGB1 in a human retinal pigment epithelial (RPE) cell line. The results show that the nuclear HMGB1 in the retinal cell is augmented by death stress and upregulation appears to be required for cell survival, whereas extracellular release of HMGB1 is evident not only in retinal cell death in vitro but also in the rat model of RD in vivo. Furthermore, the vitreous level of HMGB1 is significantly increased and is correlated with that of MCP-1 in human eyes with RD. Recombinant HMGB1 induced RPE cell migration through an extracellular signalregulated kinase-dependent mechanism in vitro. Our findings suggest that HMGB1 is a crucial nuclear protein and is released as a danger signal of retinal tissue damage. Extracellular HMGB1 might be an important mediator in RD, potentially acting as a chemotactic factor for RPE cell migration that would lead to an ocular pathological wound-healing response.

Laboratory Investigation (2009) 89, 278-289; doi:10.1038/labinvest.2008.165; published online 12 January 2009

KEYWORDS: danger signal; high-mobility group box 1 protein; photoreceptor degeneration; retinal detachment; tissue damage; wound healing

Cell death is the predominant event of degenerative tissue damage and can be a trigger that activates the immune system and repair program. Recently, there has been much interest in the pivotal role of endogenous danger signals released during cell death. ${ }^{1}$ High-mobility group box 1 (HMGB1) protein is a prototypic innate danger signal, and appears to be crucial in this context because extracellular $\mathrm{HMGB1}^{2}$ can modulate inflammation, proliferation, and remodeling, which are involved in the wound-healing process. ${ }^{3}$

HMGB1 was originally described as an abundant and ubiquitous nuclear DNA-binding protein that had multiple functions dependent on its cellular location. ${ }^{2,4}$ In the nucleus, HMGB1 binds to DNA and is critical for proper transcrip- tional regulation. It is also called amphoterin and accelerates cellular motility on the cell surface. ${ }^{5}$ HMGB1 is reported to be passively released into the extracellular milieu by necrotic cells, but not by apoptotic cells, ${ }^{6}$ or is exported actively by monocytes/macrophages ${ }^{7}$ and neural cells ${ }^{8}$ upon receiving appropriate stimuli. In damaged tissue, extracellular HMGB1 acts as a necrotic signal, which alerts the surrounding cells and the immune system. ${ }^{2}$ Although extracellular HMGB1 can contribute to normal tissue development and repair, it is also implicated in the pathogenesis of several diseases (including lethal endotoxemia, ${ }^{7}$ disseminated intravascular coagulation, ${ }^{9}$ ischemic brain, ${ }^{10}$ tumor, ${ }^{11}$ atherosclerosis, ${ }^{12}$ rheumatoid arthritis, $^{13}$ and periodontitis ${ }^{14}$ ).

\footnotetext{
${ }^{1}$ Department of Ophthalmology, Kagoshima University Graduate School of Medical and Dental Sciences, Kagoshima, Japan; ${ }^{2}$ Department of Laboratory and Vascular Medicine, Kagoshima University Graduate School of Medical and Dental Sciences, Kagoshima, Japan and ${ }^{3}$ Division of Ophthalmology, Department of Surgery, Kobe University Graduate School of Medicine, Kobe, Japan

Correspondence: Professor T Sakamoto, MD, PhD, Department of Ophthalmology, Kagoshima University Graduate School of Medical and Dental Sciences, 8-35-1, Sakuragaoka, Kagoshima 890-8520, Japan.

E-mail: tsakamot@m3.kufm.kagoshima-u.ac.jp

Received 25 August 2008; revised 9 October 2008; accepted 13 October 2008
} 
Retinal detachment (RD), the physical separation of photoreceptors from the underlying retinal pigment epithelium (RPE), is one of the main causes of visual loss. Photoreceptor degeneration due to RD is thought to be executed by apoptosis $^{15,16}$ and necrosis, ${ }^{17}$ which usually occur after tissue damage. Although retinal cell death and the following reactive responses must occur in almost all forms of retinal disease including $\mathrm{RD},{ }^{18}$ data regarding the relationship among cell death, danger, and responses in the eye, have been very limited, especially in terms of danger signals. We previously reported that HMGB1 was significantly elevated in inflamed eyes with endophthalmitis, and suggested a possible link between HMGB1 and ocular inflammatory diseases. ${ }^{19}$ On the other hand, considering the properties of HMGB1, we hypothesized that HMGB1 might have some roles in photoreceptor degeneration and subsequent damage-associated reactions in $\mathrm{RD}$.

To investigate whether HMGB1 is involved in the pathogenesis of $\mathrm{RD}$, we first examined the expression and release of HMGB1 both in a retinal cell death in vitro and in a rat model of $\mathrm{RD}$-induced photoreceptor degeneration in vivo. To focus on human $\mathrm{RD}$, we assessed the intravitreous concentrations of HMGB1 in human eyes affected by RD. Monocyte chemoattractant protein 1 (MCP-1), which was recently documented to be a potential proapoptotic mediator in $\mathrm{RD},{ }^{20}$ was also measured in the same vitreous samples. We further analyzed the effects of recombinant HMGB1 (rHMGB1) on chemotactic activity in a RPE cell line in vitro. Our findings suggest that extracellular HMGB1 is evident in eyes with $\mathrm{RD}$ as a danger signal, potentially acting as a chemotactic factor for RPE cell migration that would lead to ocular pathological wound healing.

\section{MATERIALS AND METHODS Reagents}

Full-length, LPS-free rat rHMGB1 protein, which is $99 \%$ identical to human HMGB1 and is fully functional on cells of mammalian origin, ${ }^{21}$ was purchased from HMGBiotech (Milan, Italy). Human recombinant MCP-1 (rMCP-1) was purchased from Peprotec (Rocky Hill, NJ). Rabbit polyclonal antibody against HMGB1 was provided by Shino-Test Corporation (Kanagawa, Japan). Antibodies against phosphoand total extracellular signal-regulated kinase (ERK)-1/2 were obtained from Cell Signaling Technology (Beverly, MA). U0126 was obtained from Calbiochem (La Jolla, CA).

\section{Human Vitreous Samples}

This study was approved by our institutional ethical committee (Kagoshima University Hospital), and was performed in accordance with the Declaration of Helsinki. All surgeries were performed at Kagoshima University Hospital. All patients gave informed consent before treatment. The clinical histories of all patients were obtained from their medical records. Undiluted vitreous fluid samples $(0.5-0.7 \mathrm{ml})$ were obtained by pars plana vitrectomy. Vitreous humor was collected in sterile tubes, placed immediately on ice, centrifuged to remove cells and debris, and stored at $-80^{\circ} \mathrm{C}$ until analysis.

\section{Animals}

All animal experiments were performed in accordance with the Association for Research in Vision and Ophthalmology Statement for the Use of Animals in Ophthalmic and Visual Research and the approval of our institutional animal care committee (Kagoshima University). Adult male Brown Norway rats (250-300 g; KBT Oriental, Saga, Japan) were housed in covered cages and kept at constant temperature and relative humidity with a regular 12-h light-dark schedule. Food and water were available ad libitum.

\section{Surgical Induction of RD}

Rat experimental RD was induced as described previously. ${ }^{22}$ Briefly, the rats were anesthetized with an intramuscular injection of ketamine and xylazine, and their pupils were dilated with topical $1 \%$ tropicamide and $2.5 \%$ phenylephrine hydrochloride. The retinas were detached using a subretinal injection of $1 \%$ sodium hyaluronate (Opegan; Santen, Osaka, Japan) with an anterior chamber puncture to reduce intraocular pressure. Sodium hyaluronate $(0.05 \mathrm{ml})$ was slowly injected through the sclera into the subretinal space to enlarge the RDs. These procedures were performed only in the right eye, with the left eye serving as a control. Eyes with lens injury, vitreous hemorrhage, infection, and spontaneous reattachment were excluded from the following analysis. The rats were killed at 3, 7, and 14 days after treatment, with six animals per each time point.

\section{Cell Culture}

The rat immortalized retinal precursor cell line R28, a kind gift from Dr GM Siegel (The State University of New York, Buffalo), was cultured in Dulbecco's modified Eagle's medium (DMEM) high glucose supplemented with $10 \%$ fetal bovine serum (FBS), $10 \mathrm{mM}$ non-essential amino acids, and $10 \mu \mathrm{g} / \mathrm{ml}$ gentamicin as described previously. ${ }^{23}$ The human immortalized RPE cell line ARPE-19, obtained from American Type Culture Collection (Manassas, VA), was grown in DMEM/F12 supplemented with $10 \%$ FBS, $2 \%$ penicillinstreptomycin, and $1 \%$ fungizone (all products were obtained from Invitrogen-Gibco, Rockville, MD). Cells were incubated at $37^{\circ} \mathrm{C}$ in a $5 \% \mathrm{CO}_{2}$ incubator and subcultured with $0.05 \%$ trypsin-EDTA. Subconfluent cultures were trypsinized and seeded for the following experiments. ARPE-19 cells were obtained at passage 21 and used at passages 24-30. Increased passage did not alter the following experimental results up to this passage number.

\section{Cell Viability Assay}

Cell viability was analyzed by mitochondrial respiratory activity measured using MTT (3-(4,5-dimethylthiazol-2yl)-2,5diphenol tetrazolium bromide) assay (Wako Chemicals, 
Osaka, Japan), as described previously. ${ }^{24}$ Briefly, $2 \times 10^{5} \mathrm{R} 28$ cells were cultured in 24-well plates (500 $\mu$ l medium per well) with or without hydrogen peroxide $(1 \mathrm{mM}$; Merck, Darmstadt, Germany) for $24 \mathrm{~h}$. Then the cells were incubated with MTT $(0.5 \mathrm{mg} / \mathrm{ml}$; final concentration) for $3 \mathrm{~h}$. Formazan product was solubilized by the addition of dimethyl sulfoxide for $16 \mathrm{~h}$. Dehydrogenase activity was expressed as absorbance at a test wavelength of $570 \mathrm{~nm}$ and at a reference wavelength of $630 \mathrm{~nm}$. Assays were performed in triplicate and repeated three times in independent experiments.

\section{Immunofluorescence for HMGB1 and TUNEL}

Indirect immunofluorescence was carried out as described previously, ${ }^{19,25}$ with some modifications. The eyes were harvested and fixed in $4 \%$ paraformaldehyde at $4{ }^{\circ} \mathrm{C}$ overnight. The anterior segment and the lens were removed, and the remaining eye cup was cryoprotected with $10-30 \%$ sucrose in phosphate-buffered saline. The eye cups were then frozen in an optimal cutting temperature compound (Sakura Finetech, Tokyo, Japan). Sections were cut at $8 \mu \mathrm{m}$ with a cryostat (Leica Microsystems, Wetzler, Germany). After being incubated with blocking buffer containing $10 \%$ goat serum, $1 \%$ bovine serum albumin (BSA), and $0.05 \%$ Tween- 20 for $1 \mathrm{~h}$, the slides were incubated with rabbit polyclonal antiHMGB1 antibody $(1 \mu \mathrm{g} / \mathrm{ml})$. After overnight incubation, sections were washed and probed with Alexa-Fluor 594conjugated goat anti-rabbit IgG $\mathrm{F}\left(\mathrm{ab}^{\prime}\right) 2$ fragment (Molecular Probes, Carlsbad, CA) for $1 \mathrm{~h}$. In some experiments, TUNEL co-staining was also performed according to the manufacturer's protocol (ApopTag Fluorescein In situ Apoptosis Detection kit; Chemicon, Temecula, CA) as previously described. $^{22}$ Slides were counterstained with DAPI, mounted with Shandon PermaFlour (Thermo Scientific, Waltham, $\mathrm{MA}$ ), and viewed with a Zeiss fluorescence microscope. Images were captured using the same exposure time for each comparative section. To examine the specificity of immunostaining, the primary antibody was replaced with normal rabbit IgG $(1 \mu \mathrm{g} / \mathrm{ml})$. Control slides were invariably negative under the same setting (data not shown). For all experiments, at least three sections from each eye were evaluated. To demonstrate the expression patterns of HMGB1 in retinal cells under oxidative stress in vitro, R28 cells $\left(2 \times 10^{5}\right.$ cells $/ 500 \mu \mathrm{l}$ medium per well $)$ were seeded on four-well glass coverslips and challenged with or without hydrogen peroxide $(1 \mathrm{mM})$ for $1 \mathrm{~h}$. Slides were fixed in $4 \%$ paraformaldehyde for $1 \mathrm{~h}$, permeabilized with Triton X-100, and then examined by the same methods as described above.

\section{ELISAs}

HMGB1 and MCP-1 were quantified in each human vitreous sample using commercial ELISAs; HMGB1 ELISA kit (ShinoTest Corporation) and Human CCL2/MCP-1 Immunoassay (R\&D Systems, Minneapolis, MN), according to the manufacturers' protocols. The detection limits of these kits were $0.2 \mathrm{ng} / \mathrm{ml}$ for HMGB1 and $5.0 \mathrm{pg} / \mathrm{ml}$ for MCP-1. Con- centrations below the limits were taken as zero in subsequent analyses. Each sample was run in duplicate and compared with a standard curve. All samples were assessed in a masked manner. The mean concentration was determined per sample. For in vitro study, HMGB1 levels in culture supernatants were measured by the same ELISA.

\section{Migration Assay}

Cell migration was assayed using a modified Boyden chamber assay as previously described. ${ }^{26}$ In brief, $5 \times 10^{4}$ ARPE- 19 cells resuspended in $200 \mu \mathrm{l}$ control medium (1\% FBSDMEM/F12) were seeded onto the upper compartment of the BD Falcon ${ }^{\circledR}$ culture inserts (BD Bioscience, San Jose, CA) with an $8-\mu \mathrm{m}$ diameter pore size membrane in a 24 -well companion plate. The lower chamber was filled with control medium (negative control) and those containing 50, 100, or $200 \mathrm{ng} / \mathrm{ml} \mathrm{rHMGB} 1$. Because MCP-1 was reported to display a potent chemotactic activity on RPE cells, ${ }^{27}$ a control medium containing $10 \mathrm{ng} / \mathrm{ml} \mathrm{rMCP-1}$ was used as a positive control. After 8-h incubation, cells remaining on the upper surface of the filters were removed mechanically, and those that had migrated to the lower surface were fixed with methanol, stained with Diff-Quick (Dade-Behring, Deerfield, IL), and counted in five randomly selected high-power fields $(\times 100)$ per insert. Migration index (\% of control) was calculated by dividing the number of migrating cells in the presence of chemoattractants by the cells that migrated in response to the negative control. To inhibit ERK-1/2 activity, the cells were pretreated with 1,5 , or $10 \mu \mathrm{M}$ U0126, or vehicle ( $0.1 \%$ dimethyl sulfoxide) for $30 \mathrm{~min}$, prior to the addition of rHMGB1. U0126 is an inhibitor of active and inactive MEK$1 / 2$, the MAPK kinase that activates ERK-1/2. These concentrations of U0126 and dimethyl sulfoxide had no effect on ARPE-19 cell viability determined by MTT assay in our study (data not shown) and in a previous report. ${ }^{28}$ Assays were performed in triplicate and repeated three times in independent experiments.

\section{Immunoblotting}

ARPE-19 cells $\left(5 \times 10^{5}\right)$ were subcultured on $6-\mathrm{cm}$ tissue culture dishes. Then, the cells were serum starved overnight in DMEM/F12 and stimulated with $100 \mathrm{ng} / \mathrm{ml} \mathrm{rHMGB} 1$ for the indicated times. Activation of ERK-1/2 was analyzed as described previously. ${ }^{24}$ In brief, after treatment, whole cells were lysed with SDS sample buffer and an equal volume of protein extracts was loaded onto $12 \%$ SDS-polyacrylamide gels and then transferred onto a nitrocellulose membrane. The membrane was blocked by incubation with $5 \%$ non-fat dry milk plus $1 \%$ BSA in TBST $(0.02 \%$ Tween- 20 in Trisbuffered saline, $\mathrm{pH}$ 7.4) for $1 \mathrm{~h}$ at room temperature. The membrane was then incubated with the antibody against phospho-ERK-1/2 (diluted $1 / 1000$ ) at $4^{\circ} \mathrm{C}$ overnight. The blots were subsequently probed with secondary anti-rabbit antibodies conjugated to horseradish peroxidase (diluted 1/ 3000 in TBST), and images were developed using the en- 
hanced chemiluminescence system (GE Healthcare). The membrane was stripped and reprobed with an antibody against total ERK-1/2 (diluted 1/1000).

\section{Statistical Analysis}

The vitreous HMGB1 and MCP-1 concentrations in each group were compared using the Mann-Whitney $U$-test. The correlation between HMGB1 and MCP-1 in RD samples was analyzed using a simple linear regression analysis and Spearman's rank correlation coefficient. All in vitro data are presented as mean \pm s.d. and the significance of differences between groups was determined by Student's $t$-test. $P$-value less than 0.05 was considered significant.

\section{RESULTS \\ HMGB1 is Present in Cultured Retinal Cell and Released Extracellularly by Oxidative Stress-Induced Cell Death}

We first evaluated the expression patterns and cellular distribution of HMGB1 in an R28 retinal cell line with or without oxidative stress, a known cause of neurodegeneration. ${ }^{29}$ Excessive reactive oxygen species can lead to the destruction of cellular components and ultimately induce cell death through apoptosis or necrosis. To induce oxidative stress, we used a toxic dose $(1 \mathrm{mM})$ of hydrogen peroxide, which was reported to stimulate monocytes/macrophages to release HMGB1 actively and passively. ${ }^{30}$ As shown in Figure 1a, HMGB1 immunoreactivity was stably present in the nucleus of unstimulated R28 cells, and relatively weak staining was observed in the cytoplasm. By contrast, $1 \mathrm{~h}$ after exposure to $1 \mathrm{mM}$ hydrogen peroxide, some cells seemed to present rather high levels of HMGB1 in their nucleus as well as their cytoplasm compared with those under an unstimulated condition. However, in the other cells, the nuclear HMGB1 was diminished or appeared to be released into the cytoplasm. These results indicate that the nuclear HMGB1 could be varied by death stress and be released into the cytoplasm according to the degree of stress. Hydrogen peroxide $(1 \mathrm{mM})$ treatment for $24 \mathrm{~h}$, in which about $90 \%$ of the cells lost their viability (Figure 1b), induced a massive release of HMGB1 from the cells to the cell supernatants (Figure 1c). Taken together, these findings suggested that HMGB1 could relocate from the nucleus to the cytoplasm for eventual release in dying retinal cells, and that the extracellular release of HMGB1 in the eye might be increased dependent on the extent of retinal cell death.

\section{HMGB1 is Abundantly Expressed in Rat Retina and Released after RD}

As the above findings indicated that HMGB1 was of relevance to retinal cell death, we investigated whether HMGB1 was maintained in the rat retina and how HMGB1 would vary after RD. As it was reported that HMGB1 in rat photoreceptors had a light-sensitive circadian rhythmic expression, ${ }^{25}$ we performed all animal studies on a regular time schedule, and all eyes were set to be almost equally exposed to light. As shown in Figure 2, HMGB1 immunoreactivity was well represented in sections of the normal control rat retina and, as expected, colocalized with DAPI-positive nuclei (Figure 2a, d and g). HMGB1 staining in the normal rat retina was prominent in the nuclei of ganglion cell layer, inner nuclear layer, outer nuclear layer, and RPE, and was also apparent in the photoreceptor inner segments. In particular, HMGB1 was localized in photoreceptor at the nuclear periphery, and HMGB1 levels were higher in the inner nuclear layer than the outer nuclear layer as opposed to DAPI staining, which preferred to bind to heterochromatic DNA. This was consistent with the previous report ${ }^{25}$ that HMGB1 was preferentially colocalized with euchromatin, which was often under active transcription and was stained less by DAPI. Interestingly, HMGB1 appeared to be robustly upregulated in both the photoreceptors and the other retinal cells at day 3 after RD inductions, and DAPI staining was inversely downregulated at the same time (Figure $2 \mathrm{~b}$, e and $\mathrm{h}$ ). As previous reports demonstrated that dramatic alterations of retinal gene expression occurred after $\mathrm{RD},{ }^{31}$ this high level of HMGB1 expression might be related to the active gene transcription. HMGB1 in the nucleus might be stress responsive and necessary for proper transcription after $\mathrm{RD}$ tissue damage. Afterwards, the nuclear HMGB1 expression in the photoreceptors seemed to subside at day 7 , while still clearly remained in the inner segments (Figure $2 c, f$ and i), gradually decreasing along with the thinning of the outer nuclear layer due to photoreceptor degeneration by day 14 (data not shown).

Although HMGB1 expression was increased in the photoreceptors of the detached retina at day 3, it was not homogeneous, but was rather heterogeneous. To clarify the relationship between the upregulation of HMGB1 and photoreceptor cell death, especially with DNA damage, the RD retina at day 3 was co-stained with TUNEL, which could detect apoptotic and potentially necrotic cell death by labeling the damaged DNA (Figure $3 \mathrm{a}-\mathrm{c}$ ). Previous studies indicated that HMGB1 could not be released from apoptotic cells $s^{6}$ and the apoptotic photoreceptors were prominent in this $\mathrm{RD}$ model at day 3 after $\mathrm{RD}^{22}$ We also confirmed remarkable numbers of apoptotic photoreceptors in the detached retina at day 3 (Figure 3b), and found that the early faint TUNEL-positive nuclei had relatively low levels of HMGB1 and fragmented nuclei, which were brightly stained by TUNEL, had almost no apparent HMGB1 immunoreactivity (Figure 3c), suggesting that apoptotic dying cells might lose the expression of HMGB1 to maintain the proper gene transcription. It might be indispensable for the surviving photoreceptors to maintain and/or boost the nuclear HMGB1 in RD.

In the subretinal space of $\mathrm{RD}$ at day 7, HMGB1-positive and TUNEL-negative debris could be observed (Figure 3d, arrows), which might be released by necrotic photoreceptors and/or degradated inner segments, and spread diffusely into the vitreous cavity if a retinal break was present. It was also 
a
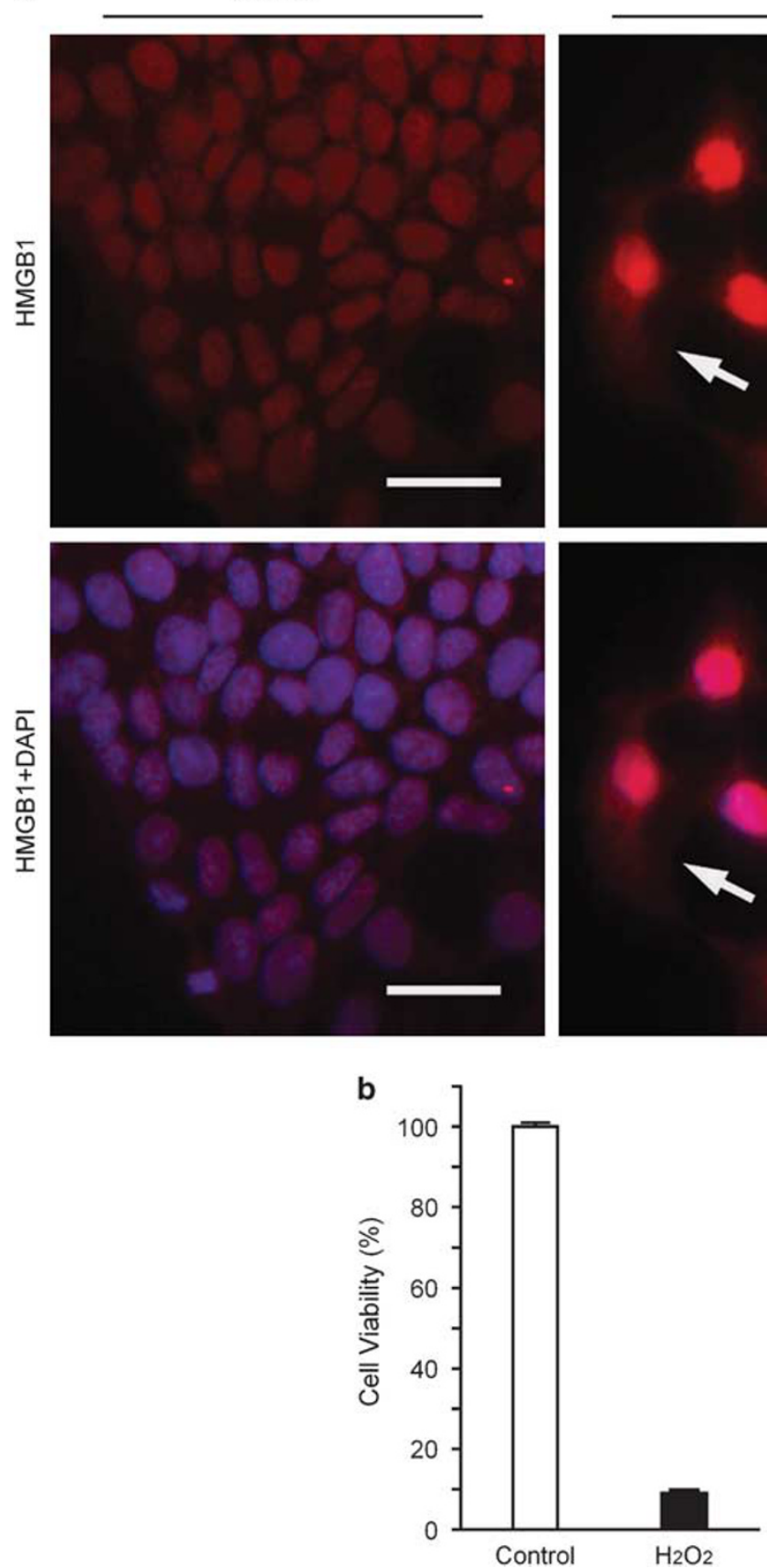

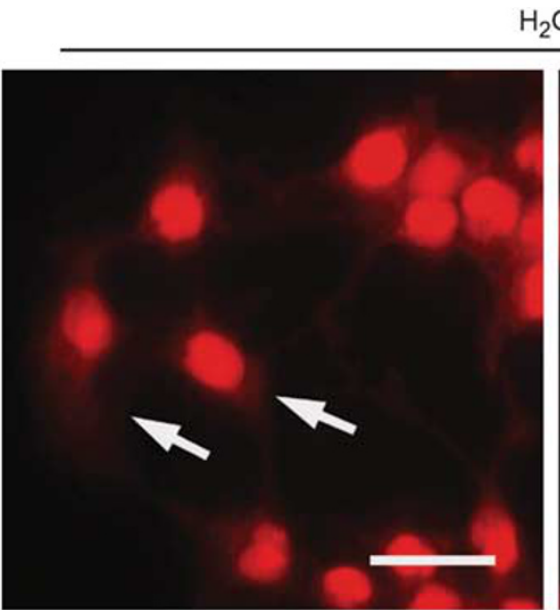

$\mathrm{H}_{2} \mathrm{O}_{2}$
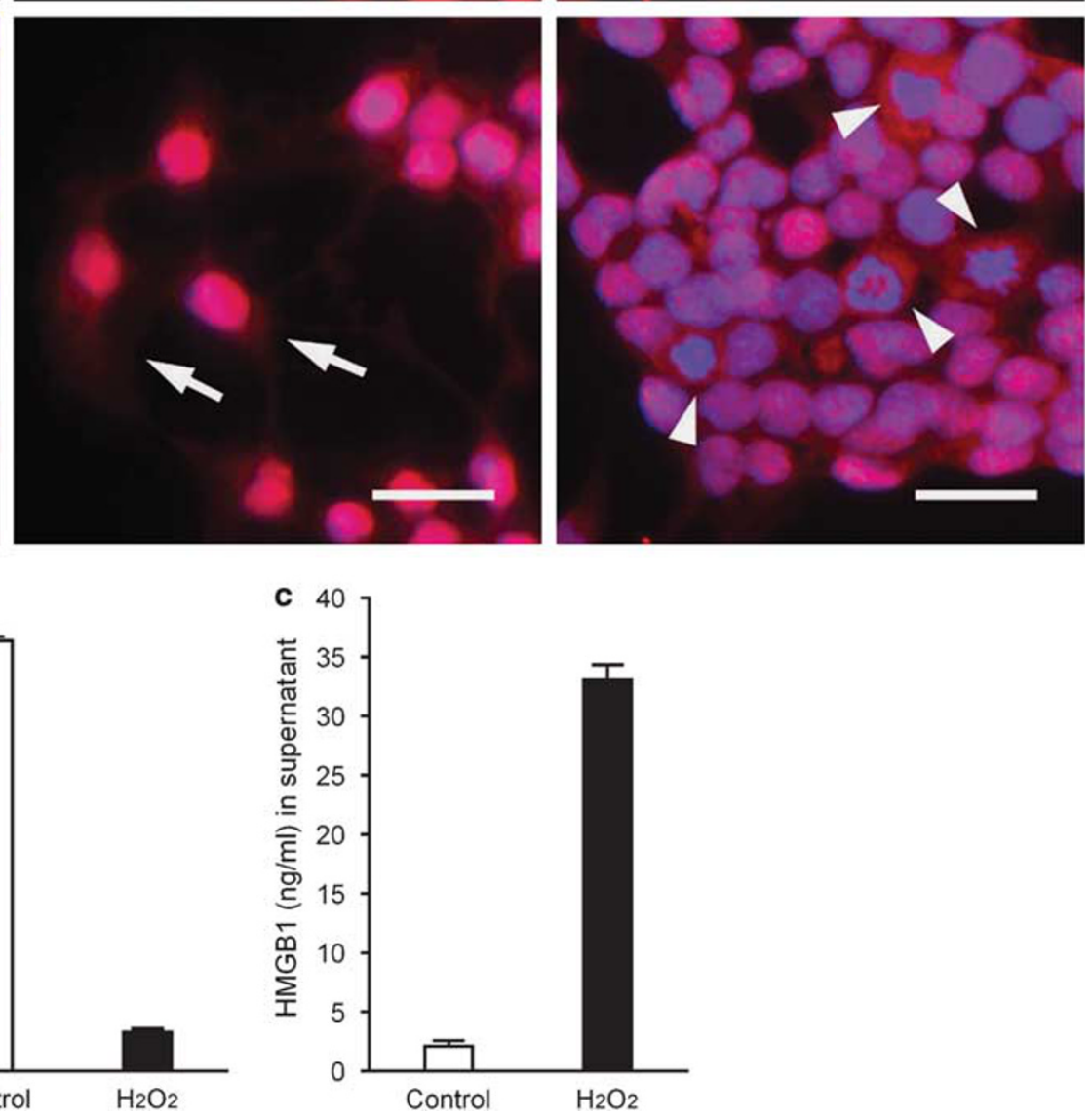

Figure 1 Release of HMGB1 from R28 retinal neuronal cells exposed to excessive oxidative stress. (a) Immunofluorescence was performed with anti-HMGB1 antibody (red) and DAPI (blue). HMGB1 is predominantly present in the nuclei of unstimulated R28 cells (left column). Some cells present robust upregulation of HMGB1 in the nuclei, as well as relocation into the cytoplasm (middle column; arrows) on $1 \mathrm{~h}$ exposure to a toxic dose of hydrogen peroxide $(1 \mathrm{mM})$. In the other cells, the nuclear HMGB1 is found to be diminished or released into the cytoplasm (right column; arrowheads). Scale bars: $20 \mu \mathrm{m}$. (b) After $24 \mathrm{~h}$ exposure to $1 \mathrm{mM}$ hydrogen peroxide, the cell viability analyzed by MTT assay is decreased to about $10 \%$ compared with the control. (c) Massive HMGB1 release into the culture supernatant was determined by ELISA after the same treatment as $(\mathbf{b})$. The data represent the mean \pm s.d. $(n=3)$. Similar results were obtained from three independent experiments.

Figure 2 Immunofluorescence analysis of HMGB1 in a rat model of RD. Representative photomicrographs of retinal sections labeled with anti-HMGB1 antibody (red; $\mathbf{a}-\mathbf{c}$ ) and DAPI (blue; $\mathbf{d}-\mathbf{f})$. Merged images $(\mathbf{g}-\mathbf{i})$ are also presented. The retinal sections were derived from the control eye $(\mathbf{a}, \mathbf{d}, \mathbf{g})$, those at 3 days (day $3 ; \mathbf{b}, \mathbf{e}, \mathbf{h}$ ), or 7 days after RD (day $7 ; \mathbf{c}, \mathbf{f}, \mathbf{i})$. Arrows point to retinal pigment epithelium $(\mathbf{a}, \mathbf{g}$ ), and arrowheads indicate subretinal macrophages $(\mathbf{b}, \mathbf{c}, \mathbf{h}, \mathbf{i})$. Note that expression of HMGB1 is augmented especially in ONL at day 3 after RD, whereas the upregulation in ONL appears to be subside by day 7 ( $n=6$ for each time point). Scale bars: $50 \mu \mathrm{m}$. INL, inner nuclear layer; IS, inner segment; ONL, outer nuclear layer. 

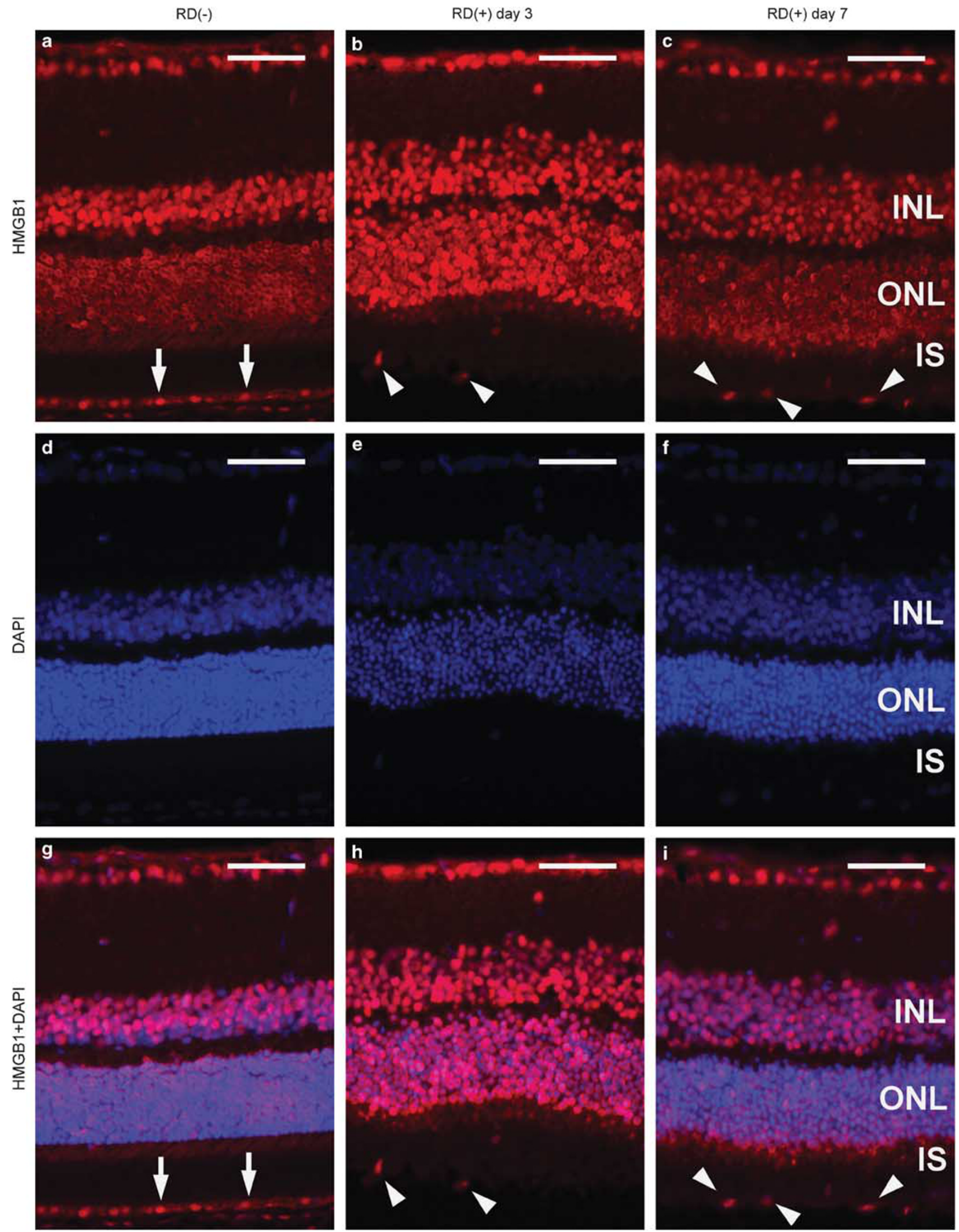
reported that macrophages migrated into the subretinal space of this RD model. ${ }^{32}$ The migrating macrophages also had abundant HMGB1 expression (Figure 3d, arrowheads), and might have released HMGB1 actively in this space. In line with these data, a large amount of extracellular HMGB1 must be present at least in the subretinal space after RD.
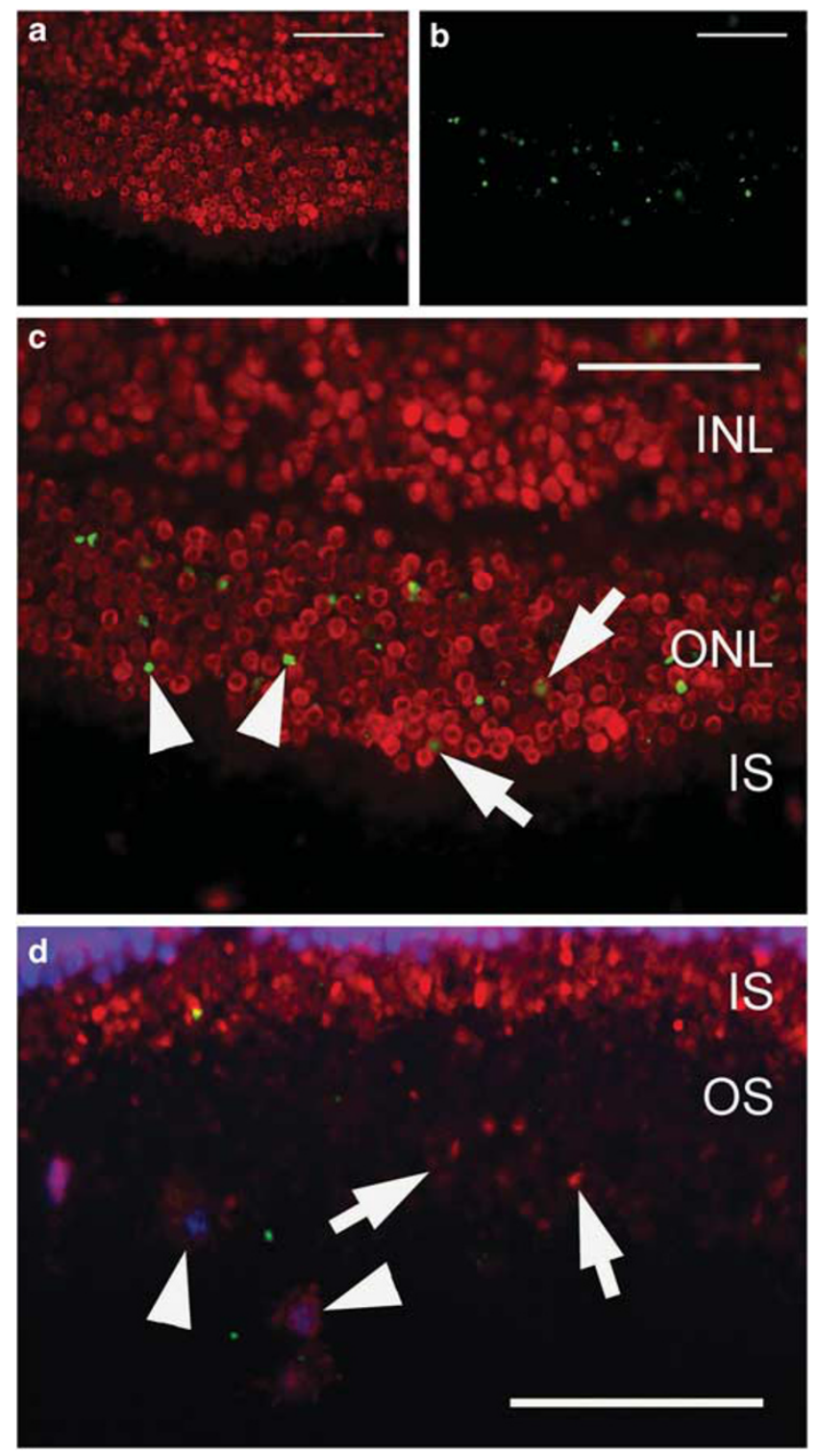

Figure 3 Expression of HMGB1 in DNA-damaged photoreceptors (a-c) and release of HMGB1 in the subretinal space (d). Representative photomicrographs of anti-HMGB1 antibody (red; a), TUNEL (green; b), and merged image (c) from rat retinal sections at 3 days after $\operatorname{RD}(n=6)$. The early faint TUNEL-positive nuclei (c; arrows) have relatively low levels of HMGB1 and the fragmented nuclei (c; arrowheads) have almost no apparent HMGB1 immunoreactivity. (d) Representative photomicrograph of a merged image of anti-HMGB1 (red), DAPI (blue), and TUNEL (green) obtained from rat retinal sections at 7 days after $\mathrm{RD}(n=6)$. HMGB1-positive and TUNEL-negative debris (d; arrows) and migrating macrophages with abundant HMGB1 expression (d; arrowheads) can be observed in the subretinal space. Scale bars: $50 \mu \mathrm{m}$. INL, inner nuclear layer; IS, inner segment; ONL, outer nuclear layer; OS, outer segment.
Vitreous HMGB1 and MCP-1 Levels in Patients with RD

The result obtained from the rat model of RD is the first evidence to our knowledge that HMGB1 is involved in RDinduced photoreceptor degeneration. Next, we tested whether extracellular HMGB1 could also be detected in human vitreous samples of RD. Samples were harvested from 35 eyes with $\mathrm{RD}$, including rhegmatogenous $\mathrm{RD}, \mathrm{RD}$ with macular hole, and atopic RD and 19 eyes with control diseases, including idiopathic epiretinal membrane and idiopathic macular hole (Table 1). The vitreous HMGB1 and MCP-1 levels were significantly higher in the eyes with $\mathrm{RD}$ than in those with control diseases (Figure 4). The median HMGB1 level was $1.4 \mathrm{ng} / \mathrm{ml}$ (range, $0-28.3$ ) in the eyes with $\mathrm{RD}$ and $0.6 \mathrm{ng} /$ $\mathrm{ml}$ (range, $0-1.3)$ in those with control diseases $(P<0.001$; Figure 4a). The median MCP-1 level was $1383.2 \mathrm{pg} / \mathrm{ml}$ (range, 39.8-5436.1) in the RD eyes and $404.4 \mathrm{pg} / \mathrm{ml}$ (range, $17.9-1168.9)$ in the control eyes $(P<0.0001$; Figure $4 \mathrm{~b})$. The vitreous concentration of HMGB1 was correlated significantly with that of MCP- 1 in the 35 eyes with RD by a simple linear regression $(r=0.593, P<0.001$; Figure $4 \mathrm{c})$ and by Spearman's rank correlation coefficient $(r=0.613$, $P<0.001)$. On the other hand, there was no significant relationship between the vitreous concentrations of HMGB1 and MCP-1 in the 19 eyes of control patients (data not shown). Although there was no significant difference, the HMGB1 levels in the eyes with proliferative vitreoretinopathy (PVR), a condition of retinal fibrosis that follows severe long-standing $\mathrm{RD}$, tended to be lower than those without PVR (Figure 4d). These findings showed that HMGB1 could be released not only in the subretinal space but also in the vitreous cavity after $\mathrm{RD}$-induced photoreceptor degeneration, and that the HMGB1 release was coincident with vitreous $\mathrm{MCP}-1$ expression.

\section{Table 1 Characteristics of the patients}

\begin{tabular}{lcc}
\hline Characteristics & $\begin{array}{c}\text { Retinal } \\
\text { detachment } \\
(n=35)\end{array}$ & $\begin{array}{c}\text { Control } \\
\text { diseases } \\
(n=19)\end{array}$ \\
\hline Age (years) & $57.3 \pm 16.3$ & $68.2 \pm 8.7$ \\
Female sex, no. (\%) & $19(54)$ & $10(53)$ \\
Patients with PVR, no. (\%) & $6(17)$ & -
\end{tabular}

Subgroups, no. (\%)

$\begin{array}{lcc}\text { Rhegmatogenous retinal detachment } & 28(80) & - \\ \text { Retinal detachment with macular hole } & 5(14) & - \\ \text { Atopic retinal detachment } & 2(6) & - \\ \text { Idiopathic epiretinal membrane } & - & 7(37) \\ \text { Idiopathic macular hole } & - & 12(63)\end{array}$

PVR, proliferative vitreoretinopathy.

Values are expressed as mean \pm s.d. Dashes denote not applicable. 

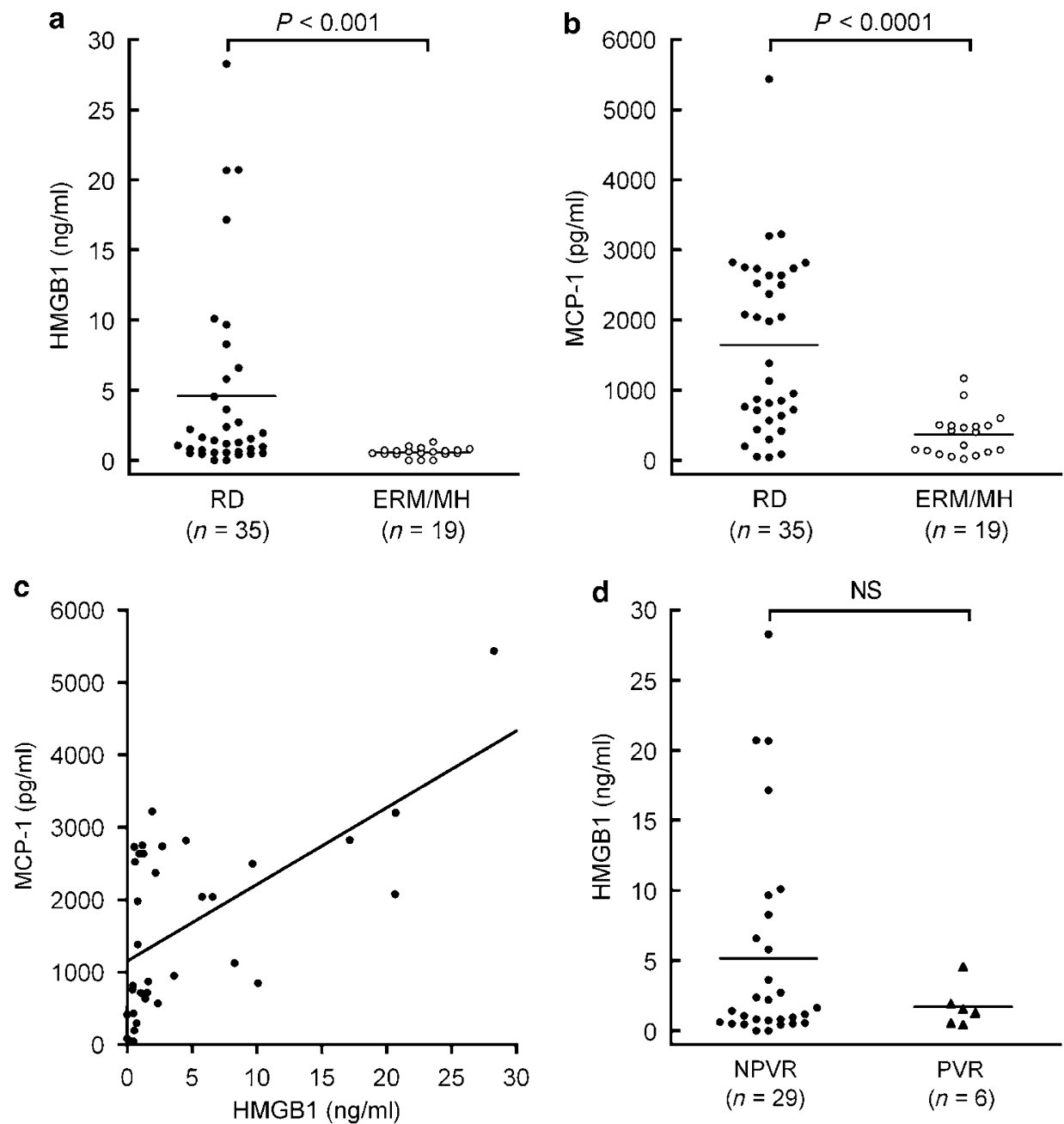

Figure 4 Vitreous levels of HMGB1 and MCP-1. The vitreous HMGB1 (a) and MCP-1 (b) levels are significantly higher in eyes with RD than in those with control diseases (idiopathic epiretinal membrane or idiopathic macular hole). Each bar indicates the average value. (c) Scatter plot for the correlation between vitreous levels of HMGB1 and MCP-1 in eyes with RD (simple linear regression, $r=0.593, P<0.001$; Spearman's rank correlation coefficient, $r=0.613, P<0.001$ ). (d) The HMGB1 levels in the eyes with PVR tend to be lower than those without PVR. ERM/MH, epiretinal membrane/macular hole; NPVR, no PVR; PVR, proliferative vitreoretinopathy.

\section{RPE Cells Respond Chemotactically to Extracellular HMGB1 through an ERK-Dependent Mechanism}

Previous reports have shown that extracellular HMGB1 is a chemoattractant for a variety of cell types. ${ }^{21,33,34}$ We investigated whether HMGB1 is also a chemoattractant for RPE cells. Extracellular HMGB1 has been reported to engage multiple receptors, including the receptor for advanced glycation end products (RAGE) and Toll-like receptors 2 and $4 .{ }^{2,4}$ In particular, RAGE has been thought to be a crucial receptor for HMGB1-induced cell migration through ERK activation. ${ }^{33}$ The expression of RAGE at the RNA and protein level was identified in human RPE ${ }^{35}$ and ARPE-19 cells ${ }^{36,37}$ in previous studies. It was also shown that the expression of RAGE and HMGB1 was colocalized in the proliferative membrane from an eye with proliferative retinal disease. ${ }^{38}$ We, therefore, performed a migration assay using modified Boyden chambers with various concentrations of rHMGB1.
The representative photographs in Figure 5 show that rHMGB1 was capable of inducing a significant level of migration (Figure 5b) above that obtained with the control medium (Figure 5a). HMGB1 stimulated the migration of RPE cells in a concentration-dependent manner with a 2.7fold maximal response at $100 \mathrm{ng} / \mathrm{ml}$ (Figure $5 \mathrm{c}$ ). This maximal response to rHMGB1 was slightly stronger than that induced by rMCP-1 $(10 \mathrm{ng} / \mathrm{ml})$. Next, we investigated whether HMGB1 induced phosphorylation of ERK-1/2 in ARPE19 cells; we stimulated cells with $100 \mathrm{ng} / \mathrm{ml} \mathrm{rHMGB} 1$ for various time periods and used western blotting with an antiphospho-ERK-1/2 antibody on whole-cell lysates (Figure 6a). Little phosphorylation of ERK-1/2 could be observed in unstimulated ARPE-19 (at $0 \mathrm{~min}$ ), but a prominent increase was detected after $5 \mathrm{~min}$ of stimulation with rHMGB1. Figure 6a shows that phosphorylation of ERK-1/2 was augmented from 5 to $60 \mathrm{~min}$ after rHMGB1 stimulation in comparison 

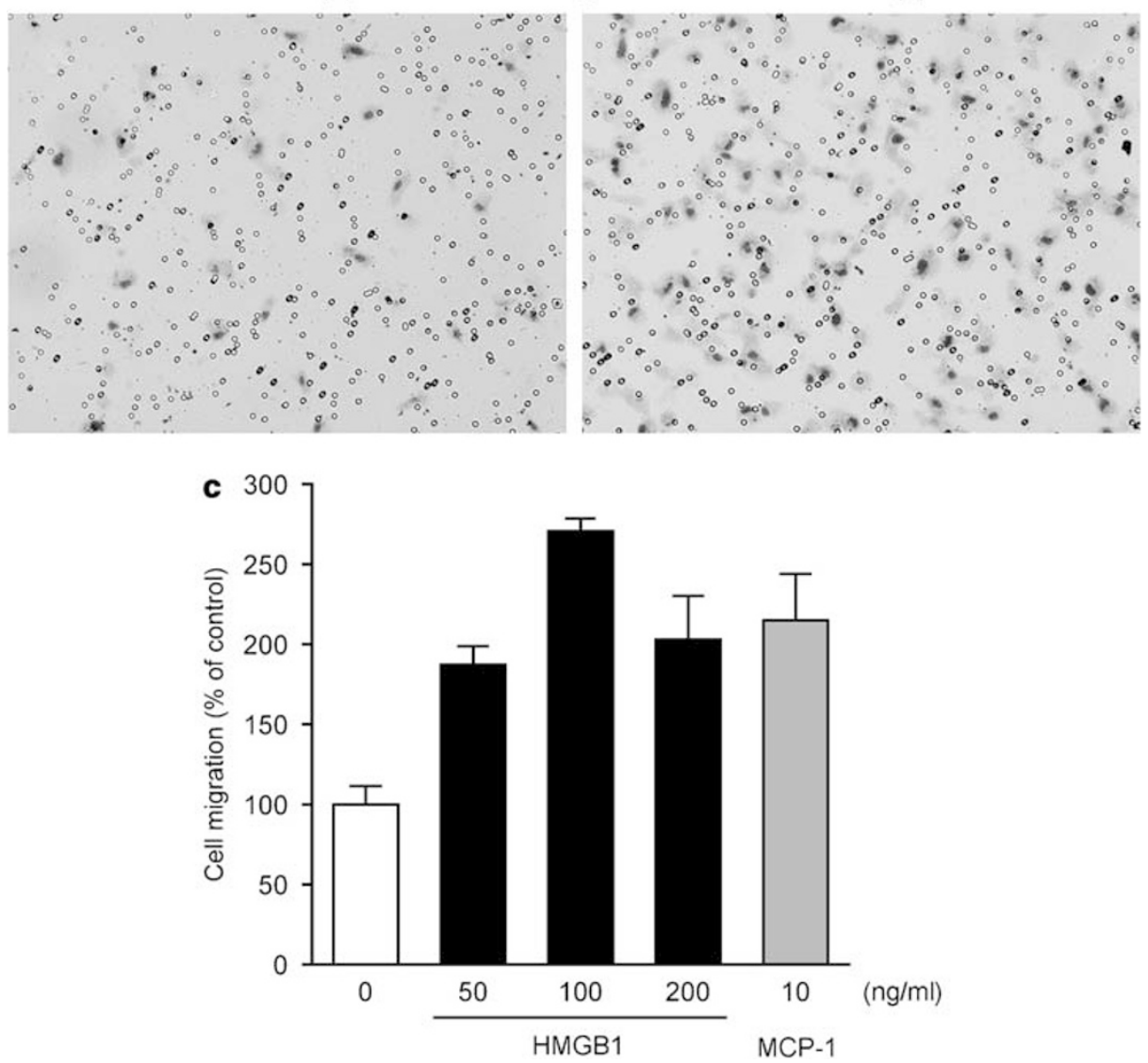

Figure 5 RPE cells migrate in response to HMGB1. Representative photographs of ARPE-19 cells stained with Diff-Quick after migration toward control medium (a) or $100 \mathrm{ng} / \mathrm{ml}$ HMGB1 (b). Original magnification: $\times 100$. (c) HMGB1 stimulated ARPE-19 cell migration in a concentration-dependent manner with a 2.7 -fold maximal response at $100 \mathrm{ng} / \mathrm{ml}$. The data represent the mean \pm s.d. $(n=3)$. All treatments increase the migratory response relative to the control $(P<0.01$ in Student's $t$-test). Similar results were obtained from three independent experiments.

with unstimulated ARPE-19 (time 0). To demonstrate that the ERK signaling induced by HMGB1 was in fact linked to the migration of RPE cells, we next inhibited ERK-1/2 and assessed cell migration to HMGB1. Pretreatment of ARPE-19 with U0126 abrogated the migration toward rHMGB1 (Figure 6b). Thus, the ERK pathway appears to play an essential role in HMGB1-induced RPE cell migration.

\section{DISCUSSION}

Our findings suggest a possible role of HMGB1 in RD, as an essential nuclear protein and a principal danger signal for photoreceptor degeneration. Using an in vitro assay of retinal cell death induced by excessive oxidative stress, we found that HMGB1 was augmented in the nucleus by the stress and released into the extracellular space during cell death. On the basis of immunohistochemical analyses of a rat model of RDinduced photoreceptor degeneration, augmentation of HMGB1 in the nucleus is also observed in vivo and appears to be crucial for the proper transcription of photoreceptors after RD. Moreover, double labeling with TUNEL reveals defects of upregulation of the nuclear HMGB1 in the DNA- damaged photoreceptors, which are presumably programmed dying photoreceptors. Therefore, we propose that the nuclear HMGB1 in the retinal cells might be critical for retinal cell survival under death stresses both in the in vivo $\mathrm{RD}$ and in vitro retinal cell death. These results for ocular HMGB1 are compatible with previous reports that HMGB1 is a vital nuclear protein and has a protective role in the nucleus. $^{2,4}$

In a previous animal study, Erickson et $a l^{17}$ reported that a loss of photoreceptors in a cat model of RD occurred due to necrosis. During studies on RD, photoreceptor degeneration after RD had been thought to be mainly caused by apoptosis. ${ }^{15,16}$ Hisatomi et al ${ }^{32}$ demonstrated the presence of apoptotic debris in the subretinal space of rat $\mathrm{RD}$. In the present study, considering our immunohistochemistry results from the same rat model of RD, so-called necrotic debris, which is HMGB1 positive and TUNEL negative, was found to be present. On the basis of the previous finding of the preferential release of HMGB1 from necrotic cells, ${ }^{6}$ this suggests that necrosis might still be a fundamental type of photoreceptor cell death after RD. 
a
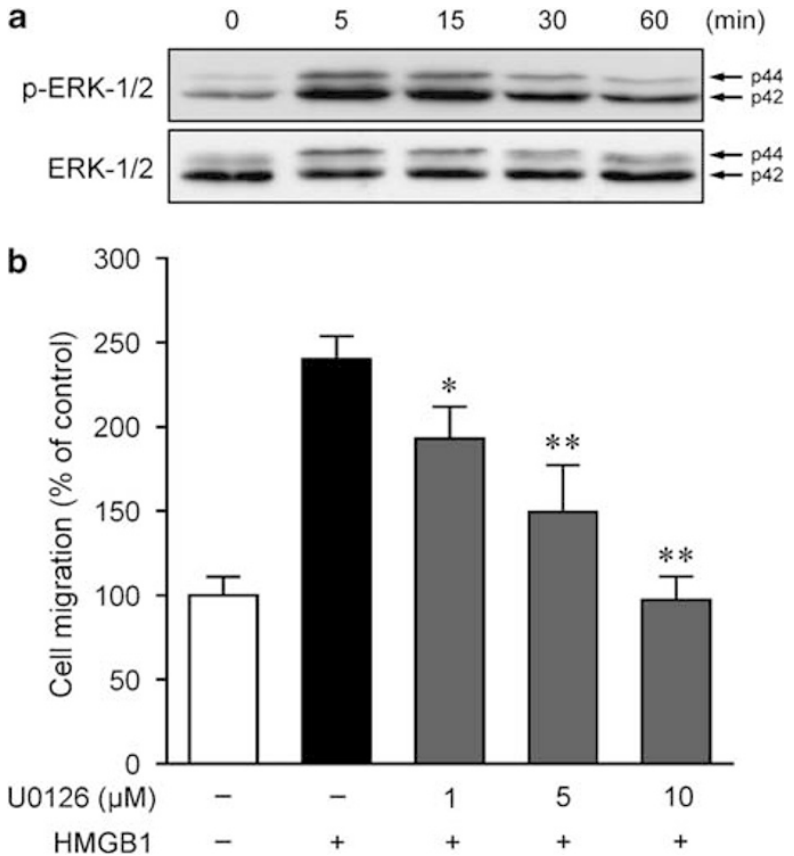

Figure 6 The phosphorylation of ERK is induced by HMGB1 and linked to HMGB1-induced migration of RPE cells. (a) ARPE-19 cells were stimulated with HMGB1 $(100 \mathrm{ng} / \mathrm{ml})$ for $5,15,30$, or $60 \mathrm{~min}$, and total cell lysates were analyzed by western blot. ERK-1/2 activation was detected with antiphospho-ERK-1/2 antibody (p-ERK-1/2). Stripped membrane was reprobed with the antibody against total ERK-1/2 (ERK-1/2). Results are representative of three independent experiments. HMGB1 augments the ERK-1/2 phosphorylation from 5 to $60 \mathrm{~min}$ after stimulation. (b) Pretreatment of ARPE-19 with U0126 inhibits the cell migration toward HMGB1 $(100 \mathrm{ng} / \mathrm{ml})$ in a dose-dependent manner. The data represent the mean \pm s.d. $(n=3)$. Similar results were obtained from three independent experiments. ${ }^{\star} P<0.05,{ }^{*} P<0.01$, compared with vehicle-treated control.

Furthermore, exploring human vitreous samples by ELISA, we found that both HMGB1 and MCP-1 are increased significantly in eyes with RD. Although MCP-1 is a well-known mediator for $\mathrm{RD},{ }^{39}$ to our knowledge, this is the first report indicating that extracellular HMGB1 might also be of relevance to human RD. HMGB1 concentration tended to be high in the eye without PVR, but not so with PVR. One possible explanation for this tendency is that HMGB1 might be sequestered and/or masked in PVR, the advanced stage of RD. HMGB1 binds tightly to heparin and proteoglycans with heparan sulfate, ${ }^{5}$ and it is also reported that such proteoglycans are abundantly present as the ocular extracellular matrix, even in $\mathrm{RD} .{ }^{40}$ Hence, these molecules might affect the HMGB1 concentration in the vitreous humor. Nevertheless, this possibility does not negate the presence of HMGB1. Considering the results obtained with the rat RD model, extracellular HMGB1 could be present at much higher levels, at least in the subretinal fluid of RD, and it might serve as a persistent signal adhering to the local damaged retina and/or surrounding matrix as previously described. ${ }^{5}$

It is also of importance that HMGB1 is significantly correlated with MCP-1 in RD vitreous. The secretion of MCP-1 might parallel the extent of photoreceptor degeneration of RD. Nakazawa et $a l^{20}$ recently suggested that MCP-1 is a potential proapoptotic mediator during $\mathrm{RD}$ through the activation of microglias and/or macrophages. In their study, Müller-glial cells were observed to upregulate MCP-1, leading to activation and increased infiltration of microglias/macrophages in the detached retina. These cells induced further photoreceptor apoptosis through local oxidative stress. Corresponding to this report, RAGE was also reported to be prominently expressed in the Müller-glial cells. ${ }^{41}$ Therefore, HMGB1 might influence MCP-1 expression through Müllerglial cells. Conversely, HMGB1 is known to be released by activated monocytes/macrophages. ${ }^{7}$ MCP-1 is a potent stimulator and chemoattractant for monocytes/macrophages, ${ }^{42}$ and these cells were observed in the subretinal space of $\mathrm{RD}$ with abundant HMGB1 expression. This would also be another possible explanation for the parallel increases of HMGB1 and MCP-1. Nevertheless, the positive correlation of these molecules indicates that cell death-related mediators might be highly orchestrated in ocular degenerative tissue damage. Several studies suggest that extracellular HMGB1 can aggravate tissue damage in neuronal tissues. ${ }^{10,43}$ In these studies, extracellular HMGB1 plays a key role in the development of neuronal injury through the induction of inflammation, microglial activation, and neuronal excitotoxicity. According to these recent reports, the presence of extracellular HMGB1 concomitantly with MCP-1 is a possible deteriorating factor for RD, in spite of its essential role in the nucleus.

PVR is one of the most threatening complications of RD. It is thought to be a reactive process to retinal injury, in other words, it is one of the wound-healing responses in the eye. RPE cells are known to be detectable in the fibrotic proliferative membranes of PVR, and play an important role in the pathogenesis of PVR. ${ }^{44}$ Thus, the effects of a molecule on PVR formation could be traced to RPE migration, at least in part. Here, we demonstrate that extracellular HMGB1 promotes RPE cell migration by chemotaxis in vitro. This result is consistent with previous reports of HMGB1-induced cell migration in various cell types, such as smooth muscle cells, ${ }^{21,33}$ fibroblasts, ${ }^{45}$ and chondrocytes. ${ }^{34}$ We also found that HMGB1 activated phosphorylation of ERK-1/2 in RPE cells and the migration induced by HMGB1 was dependent on ERK phosphorylation. The phosphorylation of ERK is associated with cell proliferation and cell migration through effects on cell-matrix contacts. ${ }^{46}$ It was also reported to be found in Müller-glial cells after RD. ${ }^{47}$ Taken together, our results suggest that extracellular HMGB1 from dying ocular cells might affect retinal cells through ERK phosphorylation and potentially serve to promote the formation of PVR, which is wound healing, but has a pathological meaning in the eye. Several new strategies for prevention of ocular fibrosis, especially targeting specific signaling pathways, have been proven to be beneficial in animal models. ${ }^{48-50}$ We propose that the identification and further characterization of danger signals, including HMGB1, would provide a novel 
perspective for better understanding the molecular pathogenesis of PVR before applying these promising therapeutic manipulations to human subjects.

It has been suggested that post-transcriptional modifications of HMGB1, such as acetylation, methylation, and phosphorylation, might influence its activity. ${ }^{51}$ Some recent reports also demonstrate that the proinflammatory activity of HMGB1 is due to combined action with other molecules. ${ }^{52}$ The present data are mostly limited to the presence of HMGB1 rather than its biological activity, and we do not address what modifications or molecules are involved in intraocular HMGB1. However, we identify for the first time that HMGB1 is evident in a typical retinal injury of human $\mathrm{RD}$, in which nuclear HMGB1 is a crucial nuclear protein and extracellular HMGB1 is a danger signal that might be required for the ocular wound-healing response. Our findings might have relevance for the underlying mechanisms of degenerative neuronal diseases. Further detailed studies will be needed to obtain more accurate knowledge and therapeutic value of HMGB1 in human diseases.

\section{ACKNOWLEDGEMENTS}

We thank Dr GM Siegel, The State University of New York, for providing R28 cells; Drs Takashi Ito, Yoko Oyama, Toshiaki Shimizu, Kazunori Takenouchi, Kiyoshi Kikuchi, Masahiro Iwata, Yuko Nawa, Yoko Morimoto, Naoki Miura, and Noboru Taniguchi for their helpful advice and technical support; Miss Nobue Uto, Tomoka Nagasato, Hisayo Sameshima, and Maiko Yamaguchi for their assistance with the experiments. This research was supported in part by a grant from the Research Committee on Chorioretinal Degeneration and Optic Atrophy, Ministry of Health, Labor, and Welfare and by a grant-in-aid for Scientific Research from the Ministry of Education, Science, and Culture of the Japanese Government.

1. Bianchi ME. DAMPs, PAMPs and alarmins: all we need to know about danger. J Leukoc Biol 2007;81:1-5.

2. Ulloa L, Messmer D. High-mobility group box 1 (HMGB1) protein: friend and foe. Cytokine Growth Factor Rev 2006;17:189-201.

3. Martin P. Wound healing-aiming for perfect skin regeneration. Science 1997;276:75-81.

4. Lotze MT, Tracey KJ. High-mobility group box 1 protein (HMGB1): nuclear weapon in the immune arsenal. Nat Rev Immunol 2005;5: 331-342.

5. Huttunen $\mathrm{HJ}$, Rauvala $\mathrm{H}$. Amphoterin as an extracellular regulator of cell motility: from discovery to disease. J Intern Med 2004;255:351-366.

6. Scaffidi P, Misteli T, Bianchi ME. Release of chromatin protein HMGB1 by necrotic cells triggers inflammation. Nature 2002;418:191-195.

7. Wang $\mathrm{H}$, Bloom $\mathrm{O}$, Zhang $\mathrm{M}$, et al. $\mathrm{HMG}-1$ as a late mediator of endotoxin lethality in mice. Science 1999;285:248-251.

8. Passalacqua $M$, Patrone $M$, Picotti $G B$, et al. Stimulated astrocytes release high-mobility group 1 protein, an inducer of LAN-5 neuroblastoma cell differentiation. Neuroscience 1998;82:1021-1028.

9. Ito $\mathrm{T}$, Kawahara K, Nakamura $\mathrm{T}$, et al. High-mobility group box 1 protein promotes development of microvascular thrombosis in rats. $J$ Thromb Haemost 2007;5:109-116.

10. Kim JB, Sig Choi J, Yu YM, et al. HMGB1, a novel cytokine-like mediator linking acute neuronal death and delayed neuroinflammation in the postischemic brain. J Neurosci 2006;26:6413-6421.

11. Campana L, Bosurgi L, Rovere-Querini P. HMGB1: a two-headed signal regulating tumor progression and immunity. Curr Opin Immunol 2008;20:518-523.

12. Inoue K, Kawahara K, Biswas KK, et al. HMGB1 expression by activated vascular smooth muscle cells in advanced human atherosclerosis plaques. Cardiovasc Pathol 2007;16:136-143.
13. Taniguchi N, Kawahara K, Yone $\mathrm{K}$, et al. High mobility group box chromosomal protein 1 plays a role in the pathogenesis of rheumatoid arthritis as a novel cytokine. Arthritis Rheum 2003;48:971-981.

14. Morimoto $\mathrm{Y}$, Kawahara Kl, Tancharoen S, et al. Tumor necrosis factoralpha stimulates gingival epithelial cells to release high mobility-group box 1. J Periodontal Res 2008;43:76-83.

15. Cook B, Lewis GP, Fisher SK, et al. Apoptotic photoreceptor degeneration in experimental retinal detachment. Invest Ophthalmol Vis Sci 1995:36:990-996.

16. Arroyo JG, Yang L, Bula D, et al. Photoreceptor apoptosis in human retinal detachment. Am J Ophthalmol 2005;139:605-610.

17. Erickson PA, Fisher SK, Anderson DH, et al. Retinal detachment in the cat: the outer nuclear and outer plexiform layers. Invest Ophthalmol Vis Sci 1983;24:927-942.

18. Vazquez-Chona F, Song BK, Geisert Jr EE. Temporal changes in gene expression after injury in the rat retina. Invest Ophthalmol Vis Sci 2004;45:2737-2746.

19. Arimura N, Ki IY, Hashiguchi T, et al. High-mobility group box 1 protein in endophthalmitis. Graefes Arch Clin Exp Ophthalmol 2008;246: 1053-1058.

20. Nakazawa T, Hisatomi T, Nakazawa C, et al. Monocyte chemoattractant protein 1 mediates retinal detachment-induced photoreceptor apoptosis. Proc Natl Acad Sci USA 2007;104:2425-2430.

21. Porto A, Palumbo R, Pieroni $M$, et al. Smooth muscle cells in human atherosclerotic plaques secrete and proliferate in response to high mobility group box 1 protein. FASEB J 2006;20:2565-2566.

22. Hisatomi T, Sakamoto T, Murata T, et al. Relocalization of apoptosisinducing factor in photoreceptor apoptosis induced by retinal detachment in vivo. Am J Pathol 2001;158:1271-1278.

23. Neekhra A, Luthra S, Chwa M, et al. Caspase-8, -12 , and -3 activation by 7-ketocholesterol in retinal neurosensory cells. Invest Ophthalmol Vis Sci 2007:48:1362-1367.

24. Biswas KK, Sarker KP, Abeyama K, et al. Membrane cholesterol but not putative receptors mediates anandamide-induced hepatocyte apoptosis. Hepatology 2003;38:1167-1177.

25. Hoppe G, Rayborn ME, Sears JE. Diurnal rhythm of the chromatin protein $\mathrm{Hmgb} 1$ in rat photoreceptors is under circadian regulation. J Comp Neurol 2007;501:219-230.

26. Hinton DR, He $\mathrm{S}$, Graf $\mathrm{K}$, et al. Mitogen-activated protein kinase activation mediates PDGF-directed migration of RPE cells. Exp Cell Res 1998;239:11-15.

27. Han $\mathrm{QH}$, Hui $\mathrm{YN}, \mathrm{Du} \mathrm{HJ}$, et al. Migration of retinal pigment epithelial cells in vitro modulated by monocyte chemotactic protein-1: enhancement and inhibition. Graefes Arch Clin Exp Ophthalmol 2001;239:531-538.

28. Glotin AL, Calipel A, Brossas JY, et al. Sustained versus transient ERK1/2 signaling underlies the anti- and proapoptotic effects of oxidative stress in human RPE cells. Invest Ophthalmol Vis Sci 2006;47: 4614-4623.

29. Klein JA, Ackerman SL. Oxidative stress, cell cycle, and neurodegeneration. J Clin Invest 2003;111:785-793.

30. Tang $D$, Shi $Y$, Kang $R$, et al. Hydrogen peroxide stimulates macrophages and monocytes to actively release HMGB1. J Leukoc Biol 2007;81:741-747.

31. Hollborn $M$, Francke $M$, landiev I, et al. Early activation of inflammation- and immune response-related genes after experimental detachment of the porcine retina. Invest Ophthalmol Vis Sci 2008;49: 1262-1273.

32. Hisatomi T, Sakamoto T, Sonoda KH, et al. Clearance of apoptotic photoreceptors: elimination of apoptotic debris into the subretinal space and macrophage-mediated phagocytosis via phosphatidylserine receptor and integrin alphavbeta3. Am J Pathol 2003;162:1869-1879.

33. Degryse $B$, Bonaldi $T$, Scaffidi $P$, et al. The high mobility group (HMG) boxes of the nuclear protein HMG1 induce chemotaxis and cytoskeleton reorganization in rat smooth muscle cells. J Cell Biol 2001;152:1197-1206.

34. Taniguchi N, Yoshida K, Ito $\mathrm{T}$, et al. Stage-specific secretion of HMGB1 in cartilage regulates endochondral ossification. Mol Cell Biol 2007;27:5650-5663.

35. Yamada $Y$, Ishibashi $K$, Ishibashi $K$, et al. The expression of advanced glycation endproduct receptors in rpe cells associated with basal deposits in human maculas. Exp Eye Res 2006;82: 840-848. 
36. Howes KA, Liu Y, Dunaief JL, et al. Receptor for advanced glycation end products and age-related macular degeneration. Invest Ophthalmo Vis Sci 2004;45:3713-3720.

37. Ma W, Lee SE, Guo J, et al. RAGE ligand upregulation of VEGF secretion in ARPE-19 cells. Invest Ophthalmol Vis Sci 2007:48: 1355-1361.

38. Pachydaki SI, Tari SR, Lee SE, et al. Upregulation of RAGE and its ligands in proliferative retinal disease. Exp Eye Res 2006;82: 807-815.

39. Elner SG, Elner VM, Jaffe GJ, et al. Cytokines in proliferative diabetic retinopathy and proliferative vitreoretinopathy. Curr Eye Res 1995;14:1045-1053.

40. Wang JB, Tian CW, Guo CM, et al. Increased levels of soluble syndecan1 in the subretinal fluid and the vitreous of eyes with rhegmatogenous retinal detachment. Curr Eye Res 2008;33:101-107.

41. Barile GR, Pachydaki SI, Tari SR, et al. The RAGE axis in early diabetic retinopathy. Invest Ophthalmol Vis Sci 2005;46: 2916-2924.

42. Matsushima K, Larsen CG, DuBois GC, et al. Purification and characterization of a novel monocyte chemotactic and activating factor produced by a human myelomonocytic cell line. J Exp Med 1989;169:1485-1490.

43. Pedrazzi M, Raiteri L, Bonanno G, et al. Stimulation of excitatory amino acid release from adult mouse brain glia subcellular particles by high mobility group box 1 protein. J Neurochem 2006;99:827-838.
44. Pastor JC, de la Rua ER, Martin F. Proliferative vitreoretinopathy: risk factors and pathobiology. Prog Retin Eye Res 2002;21:127-144.

45. Straino S, Di Carlo A, Mangoni A, et al. High-mobility group box 1 protein in human and murine skin: involvement in wound healing. J Invest Dermatol 2008;128:1545-1553.

46. Lawrence MC, Jivan A, Shao C, et al. The roles of MAPKs in disease. Cell Res 2008;18:436-442.

47. Nakazawa T, Takeda M, Lewis GP, et al. Attenuated glial reactions and photoreceptor degeneration after retinal detachment in mice deficient in glial fibrillary acidic protein and vimentin. Invest Ophthalmol Vis Sci 2007; 48:2760-2768.

48. Saika S. TGFbeta pathobiology in the eye. Lab Invest $2006 ; 86$ :106-115.

49. Saika S, Yamanaka O, Nishikawa-Ishida I, et al. Effect of Smad7 gene overexpression on transforming growth factor beta-induced retinal pigment fibrosis in a proliferative vitreoretinopathy mouse model. Arch Ophthalmol 2007;125:647-654.

50. Saika S, Yamanaka O, Sumioka T, et al. Fibrotic disorders in the eye: targets of gene therapy. Prog Retin Eye Res 2008;27:177-196.

51. Bianchi ME, Manfredi AA. High-mobility group box 1 (HMGB1) protein at the crossroads between innate and adaptive immunity. Immunol Rev 2007;220:35-46.

52. Sha Y, Zmijewski J, Xu Z, et al. HMGB1 develops enhanced proinflammatory activity by binding to cytokines. J Immunol 2008:180:2531-2537. 